\title{
EFFECTS OF THE TECHNIQUE OF CRYOPRESERVATION AND DILUTION/ CENTRIFUGATION AFTER THAWING ON THE MOTILITY AND VITALITY OF SPERMATOZOA OF OLIGOASTHENOZOOSPERMIC MEN
}

\author{
SANDRO C. ESTEVES, DEBORAH M. SPAINE, AGNALDO P. CEDENHO, MIGUEL SROUGI
}

\author{
Laboratory of Human Reproduction, Division of Urology, Paulista School of Medicine, \\ Federal University of São Paulo, Unifesp, São Paulo, SP, Brazil
}

\begin{abstract}
Objective: Comparing in human semen samples with low initial quality, the effects of 2 techniques of cryopreservation and dilution/centrifugation after thawing on the spermatic motility and vitality.

Materials and Methods: Semen samples from 15 oligo and/or asthenozoospermic individuals assisted in the infertility sector of a tertiary hospital were obtained through masturbation. The samples were divided into 2 portions of equal volume, and diluted $(1: 1 ; \mathrm{v} / \mathrm{v})$ with the cryoprotector containing glycerol (Test yolk buffer). One portion was frozen through the technique of liquid nitrogen vapor with static phases (group I - GI), while the other was frozen through a programmable biological freezer with linear speed (Planer, Kryo 10, series III) (group II - GII). The following parameters were assessed before freezing and after thawing: percentage of spermatozoa with progressive motility (Prog\%) and percentage of live spermatozoa (Vit\%). After defrosting, Prog \% was assessed before and after removal of cryoprotector diluent, in different time intervals (zero, $3 \mathrm{~h}$, and $24 \mathrm{~h}$ ). The statistical analysis has been accomplished by using the non-parametric tests of Wilcoxon and Friedman.

Results: There was significant reduction of Prog\% and Vit\% from before freezing to after defrosting in both groups, I and II ( $\mathrm{p}<0.001)$. Values of Prog\% and Vit\% were not statistically different between groups, after thawing. It has been observed a significant reduction in Prog $\%$ among portions frozen with the automated technique after dilution and centrifugation for removal of cryoprotector $(\mathrm{p}=0.006)$. After cryoprotector removal, Prog $\%$ has been kept unaltered, in both groups, during the first 3 hours of incubation, although being superior in group I $(p=0,04)$. There was a significant decrease in Prog\% after 24 hours of incubation, in both groups $(\mathrm{p}<0,01)$.

Conclusion: For human semen samples with low initial quality, freezing through vapor technique or through the automated technique showed to be equivalent in regarding recovery of live spermatozoa with progressive motility. The effects of dilution and centrifugation to remove the cryoprotector had a negative impact only in samples frozen through the automated technique. In both techniques, progressive motility is kept constant during the first 3 hours after thawing and removal of the cryoprotector, but is drastically diminished by the end of an incubation period of 24 hours.
\end{abstract}

Key words: infertility; spermatozoa; cryopreservation; sperm motility

Int Braz J Urol. 2003; 29: 133-40

\section{INTRODUCTION}

Cryopreservation of human semen is used to preserve the individual's fertility in several situations, such as before surgical procedures that can harm fer- tility, before vasectomies, or before chemotherapeutic and radiotherapeutic treatments for specific cancer cases, which generally cause germinal aplasia (1). Besides that, semen banks are used to supply donor 
semen to infertile couples (2). In artificial inseminations with donor semen, the use of cryopreserved semen is mandatory, and it should be kept under quarantine for at least 6 months, in order to avoid the presence of acquired immunodeficiency syndrome, among other sexually transmitted diseases (3). More recently, cryopreservation is been employed to store exceeding spermatozoa, aspirated from the epididymis or the testicle, for eventual use in procedures of high complexity assisted reproduction (example: ICSI intracytoplasmic sperm injection), and to store spermatozoa obtained during reconstructive microsurgeries of male genital tract (vasovasostomy and vasoepididymostomy), as additional fertility guarantees (4).

Nevertheless, even with the use of advanced cryopreservation techniques, 25 to $75 \%$ of spermatozoa die or are irreversibly harmed during the process (5). Thus, researches aiming to improve the final quality of cryopreserved human semen are welcome, for they can benefit a greater number of patients through the increase of pregnancy indexes. The objective of this study was to evaluate, in human semen samples with low initial quality, the effects of 2 cryopreservation techniques on the spermatic motility and vitality, as well as the impact of dilution/centrifugation after thawing.

\section{MATERIALS AND METHODS}

\section{Samples Collection and Seminal Analysis}

Semen samples of individuals assisted in the human reproduction sector of a university hospital were obtained through masturbation in sterile containers after sexual abstinence of 48 to 72 hours. The ethics committee for research in the institution has approved the study, and all participants signed free and informed consent. Each ejaculate sample was incubated for 30 minutes at $37^{\circ} \mathrm{C}$ to allow liquefaction. Samples were analyzed in accordance with the criteria established by OMS (6). The percentage of spermatozoa showing progressive motility [degrees $a+b$, being classified as (a), the spermatozoon with fast progressive linear movement, and as (b), slow progressive linear movement, or non-linear - Prog\%], the percentage of live spermatozoa (Vit\%), and the number of polymorphonuclear leukocytes has been evaluated. Sperm vitality has been evaluated through the eosin-negrosin supravital technique, and the number of leukocytes through the myeloperoxidase technique. The criteria for inclusion of samples in the present study were: ejaculated volume greater or equal to $2.0 \mathrm{~mL}$, sperm concentration $<20 \times 10^{6} / \mathrm{mL}$, progressive sperm motility $<50 \%$, and number of polymorphonuclear leukocytes $<1.0 \times 10^{6} / \mathrm{mL}$. At least 200 spermatozoa were analyzed from each specimen, for calculation of Prog\% and Vit\%.

\section{Cryopreservation Technique}

Each individual supplied a single sample of semen that has been divided in 2 parts of equal volume. As cryoprotective agent, a ready to use diluent has been used, commercially available, containing egg yoke at $20 \%(\mathrm{v} / \mathrm{v})$, glycerol at $12 \%(\mathrm{v} / \mathrm{v}), 85 \mathrm{mM}$ of Tris ([hydroximethyl]amino methane), 189 mM TES (n-Tris [hydroximethyl] methyl-2-amino-ethanesulphonic acid), $11 \mathrm{mM}$ of glucose, $0,25 \mathrm{mg} / \mathrm{mL}$ of streptomycin sulfate, $0.15 \mathrm{mg} / \mathrm{mL}$ of penicillin $\mathrm{pH}=$ 7,35 (Test yolk-buffer, Irvine Scientific, Santa Ana, USA). The containers containing the cryoprotective diluent were kept stored at $-20^{\circ} \mathrm{C}$, and were defrosted in water bath at $37^{\circ} \mathrm{C}$, for 20 minutes, before use. In a $15 \mathrm{~mL}$ polystyrene tube (Falcon, San Diego, USA), portions of the cryoprotector media, corresponding to $25 \%$ of volume of semen to be frozen, were added to each part at each 5 minutes, and mixed by using the aliquot mixer (Miles, Elkhart, USA). This procedure has been repeated until obtaining equal volume of diluent media and ejaculated part (proportion 1:1). The final mix was distributed into plastic sterile tubes, cylindrical with conic base, each one capable of handling $1.0 \mathrm{~mL}$ of mix (Corning, USA).

One of the parts was cryopreserved by using the liquid nitrogen vapor technique with static phases (group I - GI), while the other was frozen by using a biologic freezer with programmable linear velocities (Planer, Kryo 10, series III) (group II - GII). With the vapor technique freezing, the tubes were arranged in metallic stands appropriate to be used with nitrogen drum. A maximum of 2 tubes were arranged in each stand. Real freezing consisted of 3 consecutive steps: a) cooling phase: the metallic stands containing the 
cylindrical tubes with the sample were placed inside a freezer, with temperature adjusted to $20^{\circ} \mathrm{C}$ negative in horizontal position, and maintained inside this environment for 8 minutes, in order to reach the temperature of $+4^{\circ} \mathrm{C}$; b) freezing phase: the metallic stands containing the cylindrical tubes were transferred to the mug of the liquid nitrogen $\operatorname{drum}\left(\mathrm{N}_{2} \mathrm{~L}\right)$, being each stand placed in the vertical position, with the 2 cylindrical tubes situated in upper positions. Then, the mug was transferred to the drum containing liquid nitrogen just in its base, so that the inferior cylindrical tube was placed at $15 \mathrm{~cm}$ of the $\mathrm{N}_{2} \mathrm{~L}$ level, and the upper tube at $18 \mathrm{~cm}$. Temperature in the region occupied by the cylindrical tubes was kept around $-79^{\circ} \mathrm{C}$, checked through appropriate thermometer (Fisher, USA), and the tubes were kept inside this closed $\mathrm{N}_{2} \mathrm{~L}$ vapor environment for a 2 hours period. Freezing speed of this phase is estimated in $10^{\circ} \mathrm{C} /$ minute. Checking of $\mathrm{N}_{2} \mathrm{~L}$ level was accomplished in a systematic way before the beginning of cryopreservation process; c) storage phase: after 2 hours under $\mathrm{N}_{2} \mathrm{~L}$ vapor, the metallic stands containing the cylindrical tubes were transferred to the storage drum, being then immersed in $\mathrm{N}_{2} \mathrm{~L}$ at $-196^{\circ} \mathrm{C}$.

Automated freezing was accomplished by using a biologic freezer (Planer Kryo 10, series III, Perkasie, Pennsylvania, USA), programmed for linear reduction of temperature, in accordance with the following protocol: a) $0.5^{\circ} \mathrm{C} /$ minute, from room temperature up to $-6,0^{\circ} \mathrm{C}$; b) $10^{\circ} \mathrm{C} /$ minute, from $-6^{\circ} \mathrm{C}$ up to $-86^{\circ} \mathrm{C}$; c) immersion in liquid nitrogen at $-196^{\circ} \mathrm{C}$.

Both parts remained stored in liquid nitrogen for at least 48 hours. For sample thawing, cylindrical tubes were removed from the storage drum and kept at room temperature for 5 minutes. Next, they were transferred to a greenhouse at $37^{\circ} \mathrm{C}$, where they stayed for another 20 minutes. Removal of cryoprotective diluent was accomplished through the dilution of the thawed specimen by using modified BWW medium (HEPES-Biggers-Whitten-Whittingam, Irvine Scientific, Santa Ana, CA) (1:2, v/v), enriched with $5 \%$ of human albumin, and centrifuged for 5 minutes at $1000 \mathrm{rpm}$. The same procedure was repeated to completely remove the diluent, and the resulting sediment was re-suspended in $1.0 \mathrm{~mL}$ of modified BWW containing albumin.
After thawing, Prog\% and Vit $\%$ were evaluated, being the Prog\% evaluated before and after removal of cryoprotective diluent. After diluent removal, the Prog $\%$ was evaluated at different time intervals (zero, 3 , and $24 \mathrm{~h}$ ).

\section{Statistical Analysis}

The criterion of data normality was evaluated through the Kolmogorov-Smirnov test. Because the variables do not assume a normal distribution, the evaluation of differences of $\operatorname{Prog} \%$ and Vit $\%$, between pre-freezing and post-thawing, and between pre and post cryoprotector removal (Prog\%), was accomplished through the non-parametric test of Wilcoxon. Differences between samples frozen through manual and automated technique in relation to $\operatorname{Prog} \%$ and Vit $\%$ values were evaluated through the Friedman test. The data is expressed as median and $25 \%$ and $75 \%$ percentiles. Values of $\mathrm{p} \leq 0.05$ were considered as statistically significant. Statistical analysis was accomplished through StatSoft program, Tulsa, OK, USA.

\section{RESULTS}

The percentage of spermatozoa showing progressive motility (Prog\%) and the percentage of live spermatozoa (Vit\%), before and after cryopreservation by using the vapor and automated techniques, are expressed in Table-1. There was significant reduction of Prog\% and Vit $\%$ from before freezing to after thawing, for both groups, I and II ( $\mathrm{p}<0.001)$. Values of Prog $\%$ and Vit $\%$ were not statistically different after thawing, between groups I and II.

Post-thawing values of Prog\%, before cryoprotector removal, and at zero, $3 \mathrm{~h}$, and $24 \mathrm{~h}$ after its removal, are expressed in Table-2. There was no difference in Prog\% for groups I and II before removal of the cryoprotective diluent. It has been observed, nevertheless, stronger Prog\% reduction in aliquots frozen through the automated technique after dilution and centrifugation for removal of the cryoprotective diluent $(\mathrm{p}=0.006)$. After cryoprotector removal, the Prog\% was not altered, in both groups, during the first 3 hours of incubation, although being superior in group I $(p=0.04)$. There was a signifi- 
cant fall in Prog\% after 24 hours of incubation, for both groups $(\mathrm{p}<0.01)$.

\section{DISCUSSION}

In spite of using modern protocols for cryopreservation of human semen, sperm survival after defrosting remains unsatisfactory, and pregnancy rates are inferior to those obtained with fresh semen (7). Various studies have shown that the freezing and thawing process of human semen leads to the decrease of sperm motility (8), alterations in the pattern of penetration in the cervical mucus, decrease of penetration in hamster oocytes (9), alterations in the acrosomal structure and in the plasmatic membrane, besides the decrease in the activity of acrosomal protease (acrosin) (10). The main factors involved in the physiopathology of these events are the osmotic effects of the freezing and thawing process over the plasmatic membrane of spermatozoa, leading to alterations of permeability and cellular death (11); and the sub-lethal damage that results from a combination of factors, including: cellular dehydration and re-hydration, besides alterations in the cellular biochemistry and physiology (decrease of enzymatic activity, activation of lipidic peroxidation cascade, with consequent generation of reactive species of oxygen and oxidative damage) (12).

The fertility potential of cryopreserved semen depends, mainly on the initial quality of the sample, on the cryoprotector used, and on the freezing technique. Since the decade of 60's, cryopreservation of human semen is been routinely accomplished through the liquid nitrogen vapor technique, by using glycerol as cryoprotector (13). The best survival of human spermatozoa is obtained when: 1) the cooling velocity of room temperature up to $4 / 5^{\circ} \mathrm{C}$ is of 0.5 to $1.0^{\circ} \mathrm{C} /$ minute; 2 ) the freezing velocity of the fusion point up to $-80^{\circ} \mathrm{C}$ is around $10^{\circ} \mathrm{C} /$ minute (14), i.e., fast enough to minimize dehydration, but not so quick to cause the formation of intracellular ice. Both techniques employed in this study follow these principles, being such velocity just estimated when using the vapor technique, while with the automated technique it is possible to exactly program the freezing velocities for each process phase. Yin \& Seibel (15) checked the temperatures inside and outside of the semen samples frozen through the vapor technique, and have verified that after 10 minutes inside the vapor, the temperature of the sample reached $80^{\circ} \mathrm{C}$, which is compatible with a mean freezing velocity of $-10^{\circ} \mathrm{C} /$ minute. Nevertheless, these authors have observed a variation of $\pm 6^{\circ} \mathrm{C} /$ minute in the different points of the curve, and discrepancy of up to $30^{\circ} \mathrm{C}$ between the temperatures of vapor and sample.

As the control of temperature by using the biologic freezer is more exact, many studies have been accomplished in the intention of comparing both techniques. Although some have shown that the automated freezing preserves the quality of human semen better

Table 1 - Percentage of spermatozoa showing progressive motility (Prog\%) and percentage of live spermatozoa (Vit\%), before and after cryopreservation by using vapor and automated techniques, in 15 samples of patients having oligoasthenozoospermia.

\begin{tabular}{lcc} 
Spermatic Parameters & Prog\% Median (25\%-75\%) & Vit \% Median (25\%-75\%) \\
\hline Pre-freezing & $42.0(35.0-48.0)$ & $89.0(82.0-92.0)$ \\
Post-thawing & $8.0(3.0-24.0)$ & $46.5(39.5-61.5)$ \\
- Vapor & $10.0(2,0-28.0)$ & $58.0(35.0-71.5)$ \\
- Automated & $<0.001$ & 0.007 \\
Value of $\mathrm{P}^{1}$ & 0.84 & 0.34 \\
Value of $\mathrm{P}^{2}$ &
\end{tabular}

${ }^{1}$ Comparison of Prog\% and Vit\% values for before freezing and after thawing; ${ }^{2}$ comparison of post-thawing values of Prog $\%$ and Vit\% among aliquots frozen through the vapor and automated techniques. 
Table 2 - Post-thawing values of the percentage of spermatozoa showing progressive motility (Prog\%), before dilution and centrifugation for removal of cryoprotector, and for different incubation periods (zero, $3 h$, and $24 h$ ) after its removal, in 15 samples of patients having oligoasthenozoospermia.

\begin{tabular}{|c|c|c|c|}
\hline Removal of Cryoprotector & Vapor & Automated & Value of $\mathbf{P}^{1}$ \\
\hline Before & $8.0(3.0-24.0)^{\mathrm{a}}$ & $10.0(2.0-28.0)^{\mathrm{a}}$ & 0.84 \\
\hline \multicolumn{4}{|l|}{ After } \\
\hline zero h & $9.0(1.0-16.0)^{\mathrm{b}}$ & $5.0(1.0-17.0)^{b}$ & 0.13 \\
\hline $3 \mathrm{~h}$ & $9.0(2.0-15.0)^{\mathrm{c}}$ & $5.0(1.0-10.0)^{c}$ & 0.04 \\
\hline $24 \mathrm{~h}$ & $1.0(0.0-5.0)^{\mathrm{d}}$ & $0.0(0.0-3.0)^{d}$ & 0.62 \\
\hline${ }^{2}$ Value of $\mathrm{P}^{\mathrm{a} \times \mathrm{b}}$ & 0.22 & 0.006 & \\
\hline${ }^{2}$ Value of $\mathrm{P}$ bxc & 0.69 & 0.06 & \\
\hline${ }^{2}$ Value of $\mathrm{P}$ b or $\mathrm{Xd}$ & 0.003 & 0.002 & \\
\hline
\end{tabular}

${ }^{1}$ Comparison of Prog\% values among aliquots frozen through the vapor and automated techniques; ${ }^{2}$ comparison of Prog\% values before removal of cryoprotector, and in different incubation periods (zero, $3 \mathrm{~h}$, and $24 \mathrm{~h}$ ) after its removal.

than the vapor technique $(16,17)$, others do not confirm such benefit $(18,19)$.

Infertility is one of the sequels of cancer treatment. In approximately $50 \%$ of individuals with cancer, the seminal quality is already decreased before the beginning of the treatment. Oligoasthenozoospermia is observed in $17-77 \%$ of testicle cancer patients and in $2 / 3$ of Hodgkin disease patients (20). Many men in reproductive age having cancer are directed to cryopreservation of semen before beginning chemotherapy and/or radiotherapy. In this study, we have used samples of oligoasthenozoospermic semen, for they are the most frequently obtained from individuals that have cancer and are directed to the semen bank. We have not observed differences between the techniques in what concerns spermatic motility and vitality. Ragni et al. (21), on the other hand, have compared the 2 freezing techniques in semen samples from men that had testicle cancer and Hodgkin disease, and have verified better sperm motility with the use of automated freezing. Progressive sperm motility is one of the crucial parameters for low complexity assisted reproduction techniques to be successful, as intra-uterine insemination (22). Besides that, motility is accepted as an indicator of efficacy in the process of freezing and thawing (10).

When we compare cryo-efficiency, other factors besides those mentioned above should be taken into consideration, like cost, time, and simplicity of method execution, besides practical implications. The automated freezer is expensive and consumes 3 to 5 times more liquid nitrogen than the vapor technique. Although the execution time of the method is similar between the techniques, the automated freezing is less laborious, as the biologic freezer needs to be constantly monitored. In a practical point of view, freezing through the vapor technique is more convenient, because it allows the freezing of patients' semen at any moment in the day, being possible also to maintain the samples in the vapor at $-80^{\circ} \mathrm{C}$ during the night, without harming the final quality of the process. Besides that, any difference in the final quality of the frozen semen should be interpreted with caution, because for semen samples with very low initial quality ( $<5.0 \times 10^{6}$ motile spermatozoa), intrauterine insemination will be rarely viable to obtain pregnancy, being necessary to use in vitro fertilization associated to the intracytoplasmic sperm injection, whose chance of success does not seam to diminish even in cases in which very few motile spermatozoa are present (20).

In the majority of studies, sperm quality after freezing and thawing using a variety of techniques is evaluated immediately after thawing. Nevertheless, the cryoprotective diluent, which has toxic effects over the oocytes, should be removed in order to prepare the 
spermatozoa for intra-uterine insemination or in vitro fertilization (23). Removal of cryoprotector, which is accomplished through the dilution with culture medium and centrifugation, causes osmotic shock and even diminishes the final quality of the cryopreserved semen. In this study, the removal of the cryoprotective diluent had a negative impact over the progressive motility of spermatozoa cryopreserved through the automated technique. This harmful effect did not occur with the thawing through the vapor technique. Explanation for this finding is not clear, but it can be due to the thawing technique that has been employed. In both groups, the spermatozoa were thawed at room temperature for 5 minutes, with subsequent incubation at $37^{\circ} \mathrm{C}$. Progressive motility immediately after thawing was similar between groups, but has diminished in $50 \%$ after dilution and centrifugation with the automated technique. Other studies have shown that for freezing through the automated technique, the best sperm recovery is obtained through slow thawing, i.e., by keeping the samples under $22^{\circ} \mathrm{C}$ for at least 10 minutes $(16,18)$. Taylor et al. (16) and Verheyen et al. (18) have also observed significant reduction in the sperm motility after dilution and centrifugation for cryoprotector removal, which was, nevertheless, of the same magnitude for the manual and automated techniques. Nevertheless, the studies are not comparable, because the thawing protocol was different, and the authors assessed semen samples with high initial quality, while we have assessed samples with low initial quality.

In this study, both cryopreservation techniques allowed for progressive motility after thawing and removal of cryoprotector to be kept unaltered during the first 3 hours of incubation, being drastically diminished after 24 hours. Such fact has a practical importance, because the longevity of spermatozoa submitted to the freezing and thawing process is short, and the use of them in assisted reproduction procedures should be adequately synchronized.

\section{CONCLUSIONS}

Vapor and automated techniques are similar for oligozoospermic samples in terms of recovery of spermatozoa with progressive motility after thawing. After thawing, the longevity of spermatozoa is short, and they should be preferably used within the first 3 hours after removal of cryoprotector. The effects of dilution and centrifugation for the removal of the cryoprotector had negative impact only in samples frozen through the automated technique, and such difference can be related to the thawing speed, which will be assessed in a coming study.

\section{REFERENCES}

1. Agarwal A, Tolentino Jr MV, Sidhu RS, Ayman I, Lee JC, Thomas Jr AJ, et al: Effect of cryopreservation on semen quality in patients with testicular cancer. Urology 1995; 46: 382-9.

2. Byrd W, Bradshaw K, Carr B, Edman C, Odom J, Ackerman G: A prospective, randomized study of pregnancy rates following intrauterine and intracervical insemination using frozen donor semen. Fertil Steril. 1990; 53: 521-7.

3. Linden JV, Centola G: New American Association of Tissue Banks standards for semen banking. Fertil Steril. 1997; 68: 597-600.

4. Sharma RK, Padron OF, Thomas Jr AJ, Agarwal A: Factors associated with the quality before freezing and after thawing of sperm obtained by microsurgical epidydimal aspiration. Fertil Steril. 1997; 68: 62631.

5. Centola GM, Raubertas RF, Mattox JH: Cryopreservation of human semen. Comparison of cryopreservatives, sources of variability and prediction of post-thaw survival. J Androl. 1992; 13: 283-8.

6. World Health Organization. WHO Laboratory Manual for the examination of Human Semen and SemenCervical Mucus interaction. 4th ed, Cambridge: The Press Syndicate of the University of Cambridge. 1999.

7. Peterson EP, Alexander NJ, Moghissi KS: A.I.D. and AIDS - too close for comfort. Fertil Steril. 1988; 49: 209-10.

8. Keel BA, Webster BW, Roberts DK: Effects of cryopreservation on the motility characteristics of human spermatozoa. J Reprod Fertil. 1987; 81: 21320.

9. Critser JK, Arneson BW, Asker DV, Huse-Benda AR, Ball GD: Cryopreservation of human spermatozoa. II. Post-thaw chronology of motility and zona-free hamster ova penetration. Fertil Steril. 1987; 47: 980-4. 
10. Cross NL, Hanks SE: Effects of cryopreservation on human sperm acrosomes. Hum Reprod. 1991; 6: 127983.

11. Wastson PF: Recent developments and concepts in the cryopreservation of spermatozoa and the assessment of their post-thawing function. Reprod Fertil Dev. 1995; 7: 871-91.

12. Barthelemy C, Royere D, Hammahah S, Lebos C, Tharanne MJ, Lansac J: Ultrastructural changes in membranes and acrosome of human sperm during cryopreservation. Arch Androl. 1990; 25: 29-40.

13. Sherman JK: Low temperature research on spermatozoa and eggs. Cryobiology. 1964; 1: 103-29.

14. Henry MA, Noiles EE, Gao D, Mazur P, Critser JK: Cryopreservation of human spermatozoa. IV. The effects of cooling rate and warming rate on the maintenance of motility, plasma membrane integrity, and mitochondrial function. Fertil Steril. 1993; 60: 911-8

15. Yin H-Z, Seibel MM: Human sperm cryobanking use of modified liquid nitrogen vapor. J Reprod Med. 1999; 44: 87-90.

16. Taylor PJ, Wilson J, Laycock R, Weger J: A comparison of freezing and thawing methods for the cryopreservation of human semen. Fertil Steril. 1982; 37: 100-3.

17. MacLaughlin EA, Ford WC, Hull MG: Motility characteristics and membrane integrity of cryopreserved human spermatozoa. J Reprod Fertil. 1992; 95: 527-34.

18. Verheyen G, Pletincx I, Van Steirteghem A: Effect of frezing method, thawing temperature and post-thaw dilution/washing on motility (CASA) and morphology characteristics of high-quality human sperm. Hum Reprod. 1993; 8: 1678-84.

19. Wolf DP, Patton PE: Sperm cryopreservation: State of art. J In Vitro Fertil Embryo Transfer. 1989; 6: 3257.

20. Tournaye H, Camus M, Bollen N, Wisanto A, Van Steirteghem AC, Devroey P: In vitro fertilization techniques with frozen-thawed sperm: a method for preserving the progenitive potential of Hodgkin patients. Fertil Steril. 1991; 55: 443-5.

21. Ragni GR, Caccamo AM, Dalla Serra A, Guercilena $S$ : Computerized slow-staged freezing of semen from men with testicular tumors of Hodgkin's disease preserves sperm better than standard vapor freezing. Fertil Steril. 1990; 53: 1072-5.

22. Scammell GE, Stedronska J, Edmonds DK, White N, Hendry WF, Jeffcoate SL: Cryopreservation of semen of men with testicular tumour or Hodgkin's disease: results of artificial insemination of their partners. Lancet. 1985; 2: 31-2.

23. Graczykowski JW, Siegel MS: Motile sperm recovery from fresh and frozen-thawed ejaculates using a swimup procedure. Fertil Steril. 1991; 55: 841-3.
Received: February 10, 2003 Accepted: March 13, 2003

\author{
Correspondence address: \\ Dr. Sandro C. Esteves \\ Androfert \\ Av. Dr. Heitor Penteado, 1464 \\ Campinas, SP, 13075-460, Brazil \\ Fax: + 5519 3294-6992 \\ E-mail: s.esteves@androfert.com.br
}




\section{EDITORIAL COMMENT}

The technique of spermatozoa freezing should be very encouraged in cases of cancer, before beginning treatment, regardless of sample quality. The new techniques of assisted reproduction, especially the intracytoplasmic sperm injection (ICSI) might allow for a posterior pregnancy, even by using bad quality spermatozoa previously frozen, or with low recovery after thawing.
In this work, the observation that the process of freezing by using programmed freezer did not show any advantage over traditional freezing by using nitrogen vapor, is of a great value, for it diminishes the cost of treatment, well known as being high for the couple.

Dr. Paulo Neves Laboratory of Human Reproduction State University of Campinas, Unicamp Campinas, São Paulo, Brazil 